\title{
Los equipos directivos como líderes de la transformación educativa
}

\author{
Marlene Rocío Villegas-Gavilánez ${ }^{22}$ \\ Fe y Alegría, Ecuador \\ m.villegas@feyalegria.org.ec
}

Artículo recibido en noviembre y aprobado en diciembre 2020

\section{Resumen}

Partiendo del principio que educamos para transformar, el presente artículo hace mención del liderazgo que deben promover los equipos directivos como acompañantes de los procesos educativos en sus comunidades. Queremos apostar por directivos cuyos liderazgos generen cambios para la mejora de la calidad educativa y juntos poder vislumbrar un futuro lleno de acciones innovadoras y transformadoras que den respuestas a sus contextos. La construcción e implementación de los planes de mejora es una apuesta donde se visualiza una verdadera transformación educativa gestionada por las dimensiones de la escuela, teniendo como punto de partida el contexto, el currículo, metodologías, evaluación, roles del docente y estudiantes, la gestión educativa, la organización, los espacios de aprendizaje, la convivencia la formación y acompañamiento en esa gran aventura de soñar con una educación liberadora para las nuevas generaciones.

Palabras clave: equipos directivos, transformación educativa, acompañamiento, seguimiento, participación, innovación transformadora.

22 Apasionada por la educación que ofrece Fe y Alegría, con 19 años de experiencia en el Movimiento en los distintos niveles educativos como maestra de la UE. Mons. Oscar Romero, Vicerrectora de Unidad Educativa Fe y Alegría "La Dolorosa", Coordinadora Nacional de Bachillerato, Coordinadora de los procesos de Calidad mediados por el Sistema de Mejora de la Calidad, Coordinadora General del Área de Educación desde el 2014 hasta la actualidad y líder de la Iniciativa 09 de Innovación educativa para la transformación social a nivel de la Federación Internacional de Fe y Alegría. Coordinadora y organizadora de dos Congresos Internacionales. Máster en Liderazgo y Dirección de Centros Educativos por UNIR y Licenciada en Ciencias de la Educación, especialización Biología y Química por la Universidad Central de Ecuador. 


\section{Management teams as leaders of educational transformation}

\section{Abstract}

Based on the principle that we educate to transform, this paper mentions the leadership that management teams should promote as accompaniers to the educational processes in their communities. We want to bet on school directors whose leadership generates transformation for the improvement of educational quality and together be able to glimpse a future full of innovative and transformative actions that respond to their contexts. The construction and implementation of improvement plans is a challenge where a true educational transformation is visualized, handled by the dimensions of the school. They have as a starting point the context, the curriculum, methodologies, evaluation, roles of teachers and students, educational management, organization, learning spaces, coexistence, training and accompaniment in this great adventure of dreaming with a liberating education for the new generations.

Keywords: management teams, educational transformation, accompaniment, monitoring, participation, transformative innovation.

\section{Introducción}

"No hay mejor horizonte que aquel que se construye colectivamente" Galeano

La emergencia sanitaria a nivel global y local ha visibilizado problemáticas en los campos: educativo, social, económico, político y ambiental, lo que nos invita a poner el acento en el cuidado de las personas y sus comunidades y a reflexionar desde la educación sobre nuestras prácticas, con el fin de generar transformaciones sociales que reconozcan el contexto.

Fe y Alegría (2016) lleva 65 años de marcha sin descanso, en medio de una red que comenzó en Venezuela y que se ha ido 
expandiendo por el mundo. En Ecuador inició el camino hace más de 56 años; la utopía tiene un sentido especial en Fe y Alegría, es un reto permanente por seguir reinventando, no retroceder, ni dejar de caminar. Hoy este reto nos exige que coloquemos nuestra mirada sobre la escuela que queremos y soñamos para nuestros contextos en medio de las problemáticas que vivenciamos en el mundo, sin perder de vista la búsqueda de una innovación educativa que implique transformaciones en la vida de las comunidades.

Hoy más que nunca, estamos llamados a desestabilizarnos creativamente para poder educar personas en un mundo que nos resulta cambiante y por ello nos plantea como prioridad trastocar la forma en que se gestiona desde los equipos directivos para encaminarnos a transformar realidades. Buscamos equipos que apuesten y garanticen el derecho a una educación de calidad en sus comunidades, por ello "El modelo educativo de Fe y Alegría, implícito en su ideario, contempla la construcción de hombres y mujeres capaces de humanizar y transformar su realidad haciendo uso de sus propias capacidades y potencialidades cognitivas y afectivas" (Cabarrús, 2003, p. 9).

En este sentido, consideramos que en los contextos actuales los equipos directivos deben hacerse las preguntas: ¿Cuál sería el papel de equipo directivo como gestor del cambio y la transformación?, ¿Cuáles son los problemas y dificultades que estamos enfrentando a partir de la pandemia?, ¿qué tipo de educación debemos ofrecer a las comunidades en estos tiempos de crisis local y global?, ¿Qué tipo de formación debemos ofrecer para generar un liderazgo compartido y centrado en ámbitos pedagógicos en nuestras comunidades? Podemos ver que las preguntas revelan la existencia de dificultades y desafíos; a su vez, reconocemos desde los cuestionamientos que los problemas que hoy nos convocan pueden persistir en el tiempo si no nos detenemos a: mirar, evaluar, reflexionar, planificar, analizar el contexto para proponer nuevos caminos.

En los escenarios actuales se requieren personas que desarrollen liderazgos particulares en distintas áreas, que aunados generen resultados significativos. De esta manera se pueden equilibrar las necesidades personales, grupales e institucionales en función de ciertos 
objetivos organizacionales que generen cambios y transformaciones permanentes.

Un centro educativo no puede ser de calidad si no existe incidencia del equipo directivo en los procesos educativos. La formación de los equipos directivos es fundamental porque es necesario cambiar la cultura de la dirección unipersonal y vertical hacia un liderazgo participativo, en el que se delegan responsabilidades y funciones, buscando personas eficientes, comprometidas y críticas que puedan atender y resolver situaciones globales y específicas para conducir de manera eficaz la marcha de los centros.

Por ello, apostamos por equipos directivos que lideren procesos de transformación que busquen mejorar la calidad educativa para juntos vislumbrar un futuro colmado de acciones innovadoras que den respuestas a sus contextos y por ende a los niños, niñas y jóvenes que son nuestra razón de ser. Si somos capaces, en los centros educativos, de prepararnos para este desafío, estaremos respondiendo al motivo fundamental de la existencia de Fe y Alegría: la transformación social.

Siguiendo esta línea, el presente documento pretende ser una reflexión que oriente los planes de mejora de los centros educativos, mismos que constituyen un punto de partida con líneas de acción encaminadas a alcanzar un ideal en el que logremos equipos directivos: empoderados y competentes, con liderazgos que apuesten por su centro, donde la gestión pase por la creatividad, donde el objetivo de la transformación social sea una realidad visible. Buscamos que esta apuesta de cambio sea "con un mismo corazón y un mismo espíritu", en la hermosa aventura de soñar en una educación liberadora para las nuevas generaciones.

La propuesta es fortalecer la pertinencia, construcción, implementación, acompañamiento y seguimiento de los planes de mejora en el marco del Sistema de Mejora de la Calidad de Fe y Alegría (SMCFYA), elementos que se deben considerar para responder a las debilidades presentes en los procesos educativos (gestión, enseñanza-aprendizaje, convivencia y ciudadanía e interacción escuela comunidad), teniendo como punto de partida la realidad circundante. El 
SMCFYA implica un proceso de carácter investigativo que permita una aproximación al contexto, mismo que debe ser sistematizado a través de informes descriptivos e interpretativos, los cuales constituyen una base para formular las líneas de acción que conduzcan al centro a los resultados y metas que se planteen desde sus diversas realidades. Dicho proceso resulta de una toma de decisiones colegiada, de la participación de los distintos actores de la comunidad educativa, pues los cambios deben partir de las experiencias, necesidades y proyecciones de quienes dan vida a los espacios educativos.

\section{Desarrollo}

Fe y Alegría tiene como propósito una transformación orientada a lograr justicia educativa; con este objetivo, desde el Área de Educación Populary Promoción Social y desde el Centro de Formación e Investigación de la organización, trabajamos para crear insumos que guíen a los equipos directivos de los centros a alienarse con este gran sueño. Para ello promovemos formaciones que fortalezcan la participación y decisiones colegiadas y democráticas desde la gestión. Propiciar centros educativos que fortalezcan una cultura democrática y participativa es un modo de contribuir a la calidad educativa de un país y promover una sociedad más justa y equitativa. Se trata, por lo tanto, de mejorar el estilo de liderazgo, apostar por uno comunicativo, dialógico. Inferimos que la formación para el desarrollo profesional y personal de los equipos directivos permitirá generar espacios donde se logre una cultura de trabajo capaz de fortalecer la pertenencia de sus miembros a la institución educativa. Por este motivo se propone que los equipos directivos:

- Generen un liderazgo democrático para promover una participación real y efectiva en sus comunidades.

- Creen un ambiente de confianza en los centros educativos.

- Atiendan a las problemáticas actuales a partir de la reflexión y discernimiento de las acciones para afrontar la crisis desde miradas globales y locales.

- Generen aprendizajes significativos a partir del diálogo y las experiencias vividas

- Incorporen a la cultura institucional el trabajo en equipo y en red. 
- Mejoren la gestión institucional teniendo en cuenta los componentes de estilo de liderazgo, la optimización de los recursos y el desarrollo personal.

- Construyan un clima de participación colectiva en los diferentes actores educativos.

Freire (1997) defiende que la educación tiene como principal objetivo la transformación social; sin embargo, un cambio social no es posible solo desde la educación, en este sentido advierte:

Ni la educación es una fuerza imbatible al servicio de la transformación de la sociedad, porque yo así lo quiera, ni tampoco la perpetuación del statu quo porque el dominante así lo decrete. El educador y la educadora críticos no pueden pensar que, a partir del curso que coordinan o del seminario que dirigen, pueden transformar al país. Pero pueden demostrar que es posible cambiar. Y esto refuerza en él o en ella la importancia de su tarea político-pedagógica. (p.108)

Freire (1997) propone una educación liberadora en contraposición a la educación bancaria. La educación liberadora supone una práctica educativa que problematice nuestra realidad social, y ayude al ser humano a superar la dominación, convirtiéndose en sujeto de su propia historia. En la educación netamente tradicional que, en algunos espacios hoy en día todavía vivimos, es el profesor el que deposita sus saberes en la mente de los alumnos. En cambio, la educación liberadora opta por una pedagogía del diálogo, que se nutre de elementos como: la crítica, el amor, la humildad, la confianza, la esperanza y los sueños entre el educador y el educando. Es decir, se orienta a desarrollar la negociación cultural, la capacidad de leer, acercarse a la realidad y el desarrollo personal y comunitario.

A partir de 1981, se fue imponiendo el nombre de Educación Popular sobre el de Educación Liberadora, más tarde se generó un llamado a "reconceptualizar" la Educación Popular y a iniciar procesos de deconstrucción de teorías y prácticas, se enriqueció con aportes de las diferentes ciencias sociales como: la sociología, psicología y antropología entrando en diálogo con las pedagogías críticas. 
La Educación Popular se va comprendiendo como una intervención en el contexto desde la lectura de diversas realidades, que busca el empoderamiento de sujetos y grupos excluidos para la transformación de la realidad. Desde esta perspectiva la educación popular:

[...] hace referencia aquellos procesos políticos- pedagógicos que buscan superar las relaciones de dominación, opresión, discriminación, explotación, inequidad y exclusión. Mirada de una forma positiva se refiere a que todos los procesos educativos buscan construir relaciones equitativas y justas, respetuosas de la diversidad y de la igualdad de derechos entre las personas (Jara, 2010, p. 65).

La gestión de los equipos directivos está sujeta a la misión institucional del movimiento que se alinea con la educación popular, por ello buscamos fortalecer las transformaciones desde el contexto mediante cambios en: el currículo, metodologías, evaluación, roles del docente y estudiantes, la organización y los espacios de aprendizaje para generar incidencia en el proceso educativo, la socialización de los saberes en las comunidades; todo esto lo hacemos porque creemos que contribuye a nuestra misión, pero al mismo tiempo entendemos que existen casos particulares en los que, para aproximarnos a nuestra misión, debemos atender de forma diferenciada de modo que demos respuesta a las diferentes realidades.

Si logramos una amplia comprensión de lo que implica la gestión administrativa, veremos por qué es importante que la persona que la dirija sea quien lidere el equipo directivo y esté a la cabeza del centro educativo, pero desde una gestión democrática. Su labor no es solo la de tramitar los documentos solicitados por las dependencias del Estado o de Fe y Alegría, su trabajo es liderar y promover nuevos liderazgos, recordar cada día la misión y cómo vivirla en el cambiante mundo que experimentamos, mediar entre las familias y el sistema educativo, ser líder frente a la diversidad de situaciones coyunturales que se presentan cada día. Gestionar de manera colegiada una institución implica analizar, releer la realidad, planificar desde y para las comunidades, implementar lo planificado y hacer el acompañamiento 
y seguimiento oportuno, al mismo tiempo sistematizar experiencias significativas y saber socializarlas.

Decimos que el sueño de un equipo directivo en Fe y Alegría, se entiende de forma integral, como un espacio amplio, donde la intencionalidad es brindar una educación de calidad para todos, que sea transformadora, que nos diferencie; donde el liderazgo convierta al centro en un espacio de encuentro, comunidad, aprendizaje y organización.

Según la UNESCO (2000) existen siete prácticas de liderazgo:

Estas prácticas no son de aplicación lineal o secuencial: son más bien procesos donde el inicio de una no supone que no se pueda ir generando el despertar de la siguiente. Pueden identificarse las siguientes prácticas que favorecen la construcción del liderazgo reflexionado sobre la globalidad de los procesos de transformación educativa: Inspirar la necesidad de generar transformaciones, Generar una visión de futuro, Comunicar la visión de futuro, Promover el trabajo de equipos, Brindar orientación que desarrolle el espíritu de logro, Consolidar el avance en las transformaciones, Actualizar el aprendizaje y acumular conocimiento. (p.19)

En Fe y Alegría partimos del principio de que educamos para transformar, y por ello debemos innovar. No se trata de una innovación de mercado, como cuando se promueve un emprendimiento basado en la creación de nuevos productos y negocios desde una perspectiva capitalista, sino de una innovación que transforma, que utiliza el conocimiento orientándose al bien común. Tampoco se trata de establecer novedades, sino de contribuir de manera diferente, creativa, confiable y válida para mejorar la realidad, es decir, que promueva cambios para que los procesos educativos sean humanistas, se coloque a la persona en el centro, toquen la profundidad del ser como el sentido para generar transformaciones personales y sociales.

Para innovar nos vemos en la necesidad de reflexionar y cuestionarnos: ¿Para qué educar hoy? ¿Qué personas queremos formar 
para la vida?, ¿Qué valores, capacidades y competencias queremos fomentar en nuestros estudiantes?, ¿Qué sociedad queremos construir a partir de los problemas del contexto actual?, ¿Qué retos de transformación personal y social debemos afrontar?, ¿Qué cambios son necesarios en la educación, para poder formar las personas que queremos, y construir la sociedad y el futuro que deseamos?, ¿Cómo se articulan los problemas y demandas del entorno en las propuestas educativas de los centros?, ¿Qué acciones de mejora deben ser planteadas en los planes de mejora a partir de las problemáticas actuales?

Desde las preguntas que orientan la reflexión de nuestras prácticas, el sueño de innovación para la transformación es que nuestros centros educativos tengan una gestión más horizontal, que permitan la relación de la escuela con la comunidad, para contribuir a la toma de conciencia del rol de la escuela, la gestión debe incidir en políticas públicas para generar impacto en su barrio o comunidad; debe ser integral, permitir al estudiante poner en juego todas sus dimensiones; liberadora, donde se opte por la formación en valores humano-cristianos y ayude a mejorar nuestra praxis situando a los educadores/as en un rol de facilitadores o acompañantes para que nuestros estudiantes se conviertan en protagonistas de su propio aprendizaje.

Bajo este marco nuestra Propuesta de innovación para la transformación requiere algunos elementos a considerar:

- Más que un proceso técnico debe ser un proceso de cambio cultural, que exige voluntad política, toma de decisiones y apropiación de todos los actores educativos. Los cambios no los hacen las estructuras sino las personas.

- Un proceso de reflexión de: la gestión de los centros, del enfoque pedagógico, del proceso de enseñanza aprendizaje, del acompañamiento a las dinámicas de centro, de los procesos y vivencias personales, de los modos de convivencia e interacción con la comunidad; para a la luz de los nuevos escenarios y problemáticas del contexto actual plantear propuestas creativas que modifiquen el pensar, sentir y actuar, para transformar la realidad de injusticia y desigualdad. 
- Exige repensar todo el orden establecido y propender que su transformación, demanda cambios cualitativos significativos, no simplemente mejoras o ajustes del sistema vigente.

- Implica una intervención deliberada y planificada, y supone una aceptación y sobre todo apropiación al cambio y a la transformación de prácticas educativas.

- Recrea nuestras prácticas pedagógicas pastorales, a la luz de criterios de eficacia, eficiencia, funcionalidad, calidad, compromiso, justicia e igualdad; y desde un enfoque de educación liberadora de las potencialidades de las personas y de su entorno, enmarcadas en los principios de la Educación Popular.

- Reflexiona sobre la educación que queremos para nuestro país, identifica el propósito de nuestra innovación educativa que genere transformaciones. Este propósito implica repensar el proyecto de vida de las personas consideradas no individualmente, sino en orden a construir una ciudadanía diferente, para ello constantemente nos debemos preguntar ¿Cuál es el rol que debemos desempeñar como equipo directivo y educadores para acompañar los procesos educativos?

- El centro educativo debe pasar de enfocarse en los resultados a fijarse en los impactos que generan los procesos educativos en la vida de las personas y sus comunidades.

- Se debe tener en cuenta los tres elementos esenciales: el proyecto de vida de los estudiantes, experiencias educativas que lleven a la transformación social y una educación inclusiva de calidad.

- Hay que pasar de centros de enseñanza a centros de aprendizaje. Esto significa que el rol de los docentes no es impartir solo conocimientos, sino garantizar que sus estudiantes aprendan para la vida.

- El papel del currículo es secundario respecto al propósito central de la educación que es el fortalecimiento del proyecto de vida de los estudiantes. No significa que se deba bajar los niveles educativos, significa que hay que lograr que los estudiantes obtengan capacidades y competencias que le van a permitir ejercer una ciudadanía responsable. 
- La transformación social requiere un cambio de mirada sobre la realidad que tenemos delante y que la pandemia nos ha enseñado a entender de una manera más global e interdisciplinaria, en definitiva, debe existir un cambio en nuestros marcos mentales, de ahí que, la innovación es el instrumento o principio que nos permite pensar y ver diferente para realizar esas transformaciones personales y comunitarias que deseamos.

- Es imperativo que caractericemos el contexto y sus problemas, esto nos va a decir qué tipo de educación requerimos y con qué tipo de innovaciones educativas debemos responder a las necesidades educativas y coyunturales en este medio de cambios.

Para enriquecer estos elementos se proponen diez ideas que pueden brindar luces en torno a la innovación educativa de los centros, en este proceso es importante tener en cuenta los aportes de Casado y Díaz (2017):

1. Innovar: ¿en qué escuela? ¿Para qué? Es pensar cómo hacer una escuela comprometida con toda la comunidad educativa, que aprovecha recursos para que la educación les permita un mejor crecimiento y desarrollo. En donde la diversidad es el recurso más potente para el desarrollo integral de todos/as.

2. Visión: Imaginar horizontes, atreverse a pensar de otra manera, a romper este modelo de escuela pensado para otro momento, para otra sociedad, para otros estudiantes.

3. Ilusionar: Compartir, empoderar, imaginamos una escuela en la que todos somos protagonistas del aprendizaje. Estudiantes, familias, docentes, el barrio. Todos podemos contribuir, abriendo la posibilidad que muchos puedan aportar y enriquecer el proceso educativo.

4. Favorecer cambios: se requiere tener la apertura para generar cambios, tales como: docencia compartida, horarios más flexibles acordes a necesidades y ritmos diferentes, arquitectura apropiada y organizada, en el currículum imaginar una 
organización distinta, por ámbitos, proyectos, centros de interés, capacidades a desarrollar, competencias, interdisciplinarias, entre otros.

5. Transformar estructuras: es muy difícil que podamos favorecer la innovación sobre estructuras pensadas y creadas para otra escuela en otros momentos, deben contextualizarse y generar estructuras más horizontales.

6. Cuidar a las personas: el mejor recurso para gestionar el cambio son las personas, especialmente los educadores. No se trata de hacer más sino otras cosas y de otra manera.

7. Evaluar la innovación: la innovación debe estar vinculada a una propuesta de transformación con unos objetivos, unos tiempos, evaluación, reflexión, sistematización de prácticas, entre otros.

8. Formación: todo este proceso requerirá de acompañamiento y formación. Empezar a pensarlo de otra manera, en otros tiempos, con una docencia compartida, comprometida y de trabajo de equipo.

9. Colaboraciones y alianzas: aprovechar todos los recursos que nos rodean, generando relación escuela-comunidad, permitiendo que otros estén y participen de los espacios del centro.

10. Un futuro distinto: pensando en la escuela que queremos, en qué competencias, habilidades más que en conocimientos sin sentido, hacer de la diversidad, riqueza, una escuela más inclusiva, sin etiquetas, que ofrece oportunidades a todos y cada uno de sus estudiantes. Una escuela, en la que el rol del docente no es tanto transmitir información como cuidar y guiar ese proceso; en que la personalización del aprendizaje se convierte en el horizonte que impulsa nuestra manera de trabajar, todo ello, a través de un cambio radical en la manera en que concebimos la educación transformadora.

Estas líneas hacen referencia a las características de un centro educativo de calidad como medio para iluminar nuestro camino y mejorar 
nuestras prácticas. En este sentido, Fe y Alegría viene implementando el Sistema de Mejora de la Calidad, que propone a la escuela un proceso de evaluación, reflexión, planificación, implementación y sistematización, que se traduzca en una cultura de mejora permanente que haga posible el sueño de educar para transformar.

\subsection{Elementos del Plan de Mejora}

"No hay palabra verdadera que no sea una unión inquebrantable entre acción y reflexión"

Paulo Freire.

Para Fe y Alegría (2016) un plan de mejora no solo se define como un instrumento, sino que además es el desarrollo ordenado y sistemático de un proceso que parte de la construcción colectiva de todos los actores educativos presentes en un centro educativo, los mismos que consideran este instrumento para superar sus deficiencias, o encarar mejor aquellas debilidades y a la vez aprovechar sus fortalezas para plantearse acciones de mejora y llegar cada vez más lejos.

Por lo tanto, se propone una intervención planificada de tres años de duración, constituida por un conjunto coherente de líneas de acción que sean el camino para contribuir a la solución de uno o varios problemas de los procesos de la gestión directiva, enseñanzaaprendizaje, convivencia y construcción de ciudadanía e interacción escuela comunidad. El nombre del plan de mejora viene antecedido por procesos evaluativos, por lo que su existencia y naturaleza responde a una situación ya dada, lo que significa que su concepción, diseño, implementación, debe ayudar sobre todo a facilitar el entendimiento y continuidad del proceso iniciado, no a complejizar o confundir a los actores que están en los centros educativos en la dinámica de la apropiación.

"Pensar un plan, implica sentarse juntos a imaginar qué queremos, hacia dónde vamos, con qué contamos, en qué tiempo y lugar lo vamos a hacer, con qué posibilidades se cuentan, qué alianzas hay que hacer" (Gyssels, 2011, p.14). Este plan tiene unas características propias que surgen de: 
- Un proceso de evaluación, lectura y mirada del contexto y de la reflexión donde participa toda la comunidad educativa. Parte de un problema definido por la comunidad educativa, generado en espacios de reflexión de todos los actores como equipos directivos, docentes, estudiantes, familia y comunidad, sin perder de vista el sueño o una meta de lo que se quiere transformar en tres años, que apuesta por la innovación transformadora, entendida no solo como un cambio, sino como la posibilidad de aportar con ideas creativas a la solución de los problemas existentes en la sociedad y en particular aquellos que surgen vinculados a los procesos educativos. En el terreno pedagógico se trata de acciones que afecten puntual y directamente a todos los procesos vistos desde la integralidad, pero sólo cuando aquellas tocan también el terreno social aportando a su transformación.

- Está proyectado a tres años, por lo tanto, sus acciones son en proceso y se articulan con propuestas direccionadas por el Ministerio de Educación.

- Da respuesta y está articulado con la propuesta pedagógica el proyecto educativo institucional.

- Permite realizar seguimiento a la gestión tanto directiva como pedagógica de los centros educativos.

- Está basado en algunos planteamientos básicos que define y describe muy claramente de dónde parte; a dónde quiere llegar; cuál es el camino; qué resultados, indicios y acciones se van a alcanzar.

El papel protagónico de los equipos directivos como acompañantes de sus procesos y por ende de la implementación de los planes de mejora, se convierten en un eje facilitador de experiencias que implica situarse al lado del otro como compañero de camino, estableciendo relaciones profundas de diálogo y amistad, de compañía que permiten crecer y compartir recíprocamente. El acompañamiento busca fortalecer a las instituciones educativas con acciones en proceso para cada estamento de la comunidad educativa en función de generar 
reflexión sobre la práctica, el acompañamiento se realiza en varios niveles y dimensiones partiendo de la organización de los centros. Por ello el liderazgo ayuda a la toma de decisiones que no es nunca una cuestión de una sola persona, implica jugarse por las personas y jugarse por la educación, por supuesto con la pasión que distingue al educador y la educadora que se mueve por el espíritu y el deseo de servir.

Su finalidad de transformación está presente a lo largo de todo el proceso, parte de una situación inicial identificada y caracterizada, contemplando uno o varios problemas que afectan a la institución educativa, y cuyo objetivo es avanzar hacia el compromiso de la transformación social y construcción de sujetos líderes y empoderados de sus propios procesos.

Los planes de mejora se conciben como consecuencia de la predisposición y voluntad colectiva por transformar e innovar los procesos que dan vida a la institución educativa impulsados por la vivencia de una evaluación (interna y/o externa).

En ese marco el Plan de Mejora puede significar o se entiende como:

- Un componente central de los procesos que vive el centro educativo a partir de sus transformaciones educativas como el currículo, metodologías, evaluación, roles del docente y estudiantes, la organización y los espacios de aprendizaje para generar incidencia en el proceso educativo desde socializar los saberes en las comunidades.

- Un camino que se orienta a la innovación para la transformación educativa

- Un motor que impulsa la cultura de mejora permanente en la búsqueda del derecho a una educación de calidad para nuestras comunidades.

- Una guía que posibilita cambios a nivel estructural y organizativo y que fomenta la sistematización y socialización de experiencias significativas. 
En Fe y Alegría hemos pasado de una lógica de planificación de objetivos a una de procesos y resultados que son flexibles en el tiempo, los elementos que constan en el plan de mejora son los siguientes:

- El problema, como un fenómeno, situación o hecho real, evidente, comprobable que afecta negativamente a un contexto escolar o que permite encarar las debilidades para convertirlos en fortalezas, debe responder netamente a los procesos educativos (gestión directiva, enseñanzaaprendizaje, convivencia y ciudadanía e interacción escuela comunidad), es clave plantearse las siguientes preguntas que puedan generar reflexión en los actores, ¿cuál es la situación negativa o hecho real del problema identificado?, ¿cuál es la característica o variable más destacada del problema?, ¿quiénes son afectados por esta situación?. Resulta imprescindible socializar constantemente a todos los actores de la comunidad educativa para que conozcan, comprendan y se apropien del o los problemas de su centro y comunidad.

- La meta, es el sueño que tenemos para nuestro centro educativo, es lo que queremos lograr en tres años. Es un sueño que no necesariamente se logra con un plan de mejora, sino que nos permite acercamos a la escuela que queremos en perspectiva de innovación transformadora. La meta se caracteriza por ser desafiante, motivadora para la acción y sobre todo para la transformación.

- La línea de acción, es aquella que permite visualizar el camino a recorrer y la que posibilita una aproximación al sueño de transformación educativa, responde al cómo y está relacionada con el problema o problemas definidos por los actores del centro educativo, es importante tener claro el camino a recorrer, la disponibilidad que el centro educativo muestra para la innovación transformadora, claridad de la meta para la toma de decisiones de cómo acercarnos a ella, la descripción debe ser de manera sustantiva, es clave, la apropiación de los principios de la educación popular. 
- Los resultados, en el plan de mejora hacen referencia a los logros o productos concretos, visibles, tangibles; los puntos de llegada en los cuales se apoya el proceso y camino a recorrer hacia la consecución de la meta, los criterios para su formulación dependerán de la descripción de la población receptora y de la descripción de la situación de cambio, la cual será medible y evaluable, es importante cuando se construye un resultado empezar por el año tres, en la medida en la que se va implementando puede tener ajustes.

- Los indicadores de resultado, se consideran como pistas, hitos y evidencias del proceso que está desarrollándose, son señales que nos acercan al resultado, permiten la medición en la reflexión de las prácticas educativas, además hacer posible la evaluación y seguimiento de lo que se ha propuesto.

Los indicadores deben ser concretos y específicos, cuantificables u objetivamente verificables y sobre todo pertinentes, y se debe tomar en cuenta que establecen los aspectos que caracterizan a los resultados.

Los indicadores de resultado miden los logros o efectos del proceso vivido y del sueño que se desea alcanzar; es decir permiten establecer si las acciones ejecutadas contribuyendo a lograr los resultados planteados y si se avanzó en la consecución de la meta. Los elementos de un indicador de resultado son: la cantidad (porcentaje a obtener), la población objeto, el contenido de la mejora y su temporalidad (año lectivo).

- Indicadores de Proceso: brindan señales para hacer seguimiento a los procesos que se desea vivir a lo largo de la implementación de los planes de mejora; dan pistas para generar ajustes a lo largo de la implementación, tienen dos partes fundamentales que son sujeto (población) y el contenido para la mejora permanente de las prácticas.

- Las acciones: son el macro de actividades que generan nuevos saberes y aprendizajes, estas deben ser pensadas en función de las problemáticas identificadas y de la innovación transformadora que se quiere generar; deben 
darse en proceso para lograr los resultados esperados, las acciones deben pensarse en función de: lo que voy a mantener (porque dieron resultados y son fortalezas), lo que voy a transformar (porque si bien he avanzado sigue manteniéndose como una debilidad en el centro) y lo que voy a innovar (en el caso de presentar debilidad y no haber avanzado). Debemos preguntarnos constantemente ¿Qué acciones debo plantear para fortalecer los procesos educativos de mi centro según los resultados explícitos en el plan de mejora?

La gráfica resume los elementos que se consideran en los planes de mejora de centros educativos.

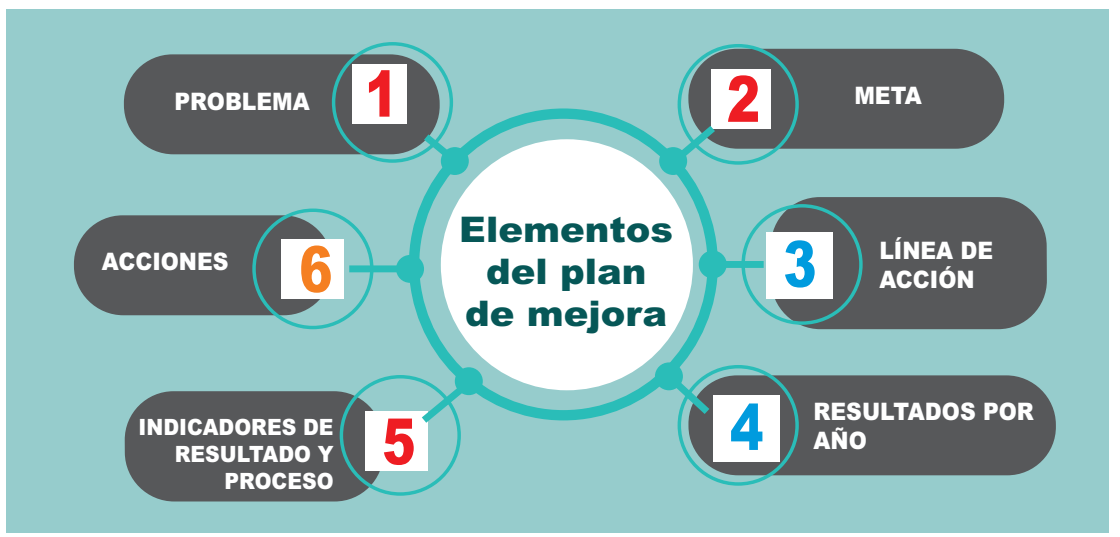

Fuente: elaboración propia

Para que esta propuesta de construcción e implementación de los planes de mejora cobre vida en los centros educativos, se deben generar unas condiciones para cumplir con el sueño de transformación según el propio Sistema de Mejora de la Calidad de Fe y Alegría. Siguiendo a Riveros (2007):

- La socialización: Entendida como las acciones en proceso con espacios abiertos y flexibles que un centro educativo realiza para que los miembros de su comunidad educativa vayan adquiriendo la cultura permanente de mejora, conozcan y se empoderen de los procesos educativos. 
- La formación: Son todas aquellas acciones de aprendizaje encaminados al logro de desarrollo de habilidades de la comunidad educativa para la comprensión de los elementos y logros de transformación. La formación debe ser innovadora, organizada y sistematizada a través de experiencias planificadas, con la intencionalidad de generar una nueva cultura de transformación en los miembros de la comunidad educativa.

- La participación: Se entiende por participación aquellas acciones en las cuales personas de la comunidad educativa toman parte activa en un espacio, posicionándose y haciendo propuestas en busca del bien común. Esta condición requiere resaltar logros a lo largo del proceso democratizador del sistema educativo. Es el ejercicio del poder que cada uno desarrolla, para transformar la escuela y las acciones que en ella se desarrollan. También es la intervención en las decisiones y relacionadas con la planificación, la actuación y la evaluación que ha de tener lugar en el centro educativo y en el aula.

- La organización: El centro educativo se organiza en un sistema de actividades conscientemente coordinadas, con buenos procesos de comunicación entre los integrantes de la comunidad, que les permiten actuar para obtener un objetivo común. El centro educativo se organiza a través de la constitución de diversos equipos que hacen posible la mejora de calidad. La organización les hace llevar de manera ordenada actas, memorias, registros de las diferentes acciones que se realizan para obtener el resultado esperado.

- La articulación: Entendida como la capacidad del centro educativo de hacer alianzas o uniones entre los procesos, programas y proyectos que están desarrollando. El programa de mejora se convierte en el eje articulador de las acciones que desarrolla el centro educativo. La articulación implica un proceso de toma de decisiones en el diseño de estrategias intrainstitucionales tendientes a desarrollar acciones unificadas entre los diferentes programas o proyectos. 
- El seguimiento: Es una actividad continua que provee información sobre el progreso de cada una de las líneas de acción presentadas en el plan de mejora, mediante la comparación de avances periódicos y metas predefinidas. Proporciona elementos para los ajustes necesarios.

- El acompañamiento: Es un eje facilitador de experiencias que implica situarse al lado del otro como compañero de camino, estableciendo relaciones profundas de diálogo y amistad, de compañía que permiten crecer y compartir recíprocamente. El acompañamiento, busca fortalecer a las instituciones educativas con acciones en proceso para cada espacio de la comunidad educativa para generar reflexión sobre la práctica.

\section{Conclusiones}

- El rol del equipo directivo como líder acompañante de los procesos educativos, implica situarse al lado del otro como compañero de camino, estableciendo relaciones profundas de diálogo y cercanía, que permiten crecer y compartir recíprocamente. El acompañamiento busca fortalecer las instituciones educativas con acciones en proceso para generar reflexión y empoderamiento sobre la práctica, desde un liderazgo comunicativo, abierto y flexible.

- El liderazgo que apuesta por la transformación tiene que ver con la toma de decisiones, no de una sola persona, sino colectiva, implica jugarse por las comunidades y por la educación que queremos, por ello es fundamental la vivencia de la gestión colegiada y el trabajo de equipo, puesto que aquello implica analizar el contexto para releer la realidad, planificar desde y para las comunidades, implementar lo planificado, reflexionar y discernir los procesos educativos que hagan posible este gran sueño de educar para transformar.

- La innovación transformadora no solo pensada como el cambio de una cosa en otra diferente, sino como la 
posibilidad de aportar con ideas creativas a la solución de los problemas existentes en la sociedad y el contexto, en particular aquellos que surgen vinculados a los procesos educativos de la gestión directiva, enseñanza aprendizaje, convivencia y ciudadanía e interacción de la escuela y la comunidad, generan innovación sólo cuando tocan también el terreno social aportando a su transformación.

- La participación en la toma de decisiones es la clave en la gestión de un centro educativo por lo que comprometerse de lleno con los principios de igualdad y equidad para todos implica también transformar las formas en las que tradicionalmente se han tomado las decisiones y los círculos de participación a los que se les ha dado acceso a los actores educativos como parte activa en un espacio donde tomen posicionamiento y desarrollan propuestas en busca del bien común.

- El diseño del plan de mejora nos invita a imaginar la transformación educativa a largo plazo, tiene en cuenta componentes centrales de los procesos que vive el centro: el currículo, metodologías, evaluación, roles del docente y estudiantes, la organización y los espacios de aprendizaje para generar incidencia en el proceso educativo desde socializar los saberes en las comunidades. Contempla la problemática encontrada, el sueño de transformación, el camino a seguir, los resultados, indicadores y las acciones que hacen posible las opciones éticas, políticas y pedagógicas para la mejora continua de la calidad educativa.

\section{Bibliografía}

Cabarrús, C. (2003). Ser persona el plenitud. La formación humana desde la perspectiva ignaciana. Caracas: Federación Internacional Fe y Alegría y Fundación Santa María.

Casado, G. y Díaz, M. (2017). Diez ideas en torno a la innovación en los centros. Recuperado de: http://www.eduforics.com/es/diez-ideastorno-la-innovacion-los-centros/. 
Fe y Alegría, (2016). Horizonte Pedagógico Pastoral Fe y Alegría Ecuador. Recuperado de http://www.feyalegria.org.ec/images/biblioteca/ Horizonte-Pedagogico-Pastoral-Fe-y-Alegria-Ecuador.pdf

Freire, P. (1997). Pedagogía de la autonomía: saberes necesarios para la práctica educativa. Madrid: Siglo XXI Editores.

Gyssels, S. (2011). Planificar el cambio. Bogotá: Federación Internacional de Fe y Alegría.

Jara, O. (2010). Educación Popular y cambio Social en América Latina. Oxford University Press and Comunity Development Journal. Recuperado de https://dalbandhassan.files.wordpress. com/2011/04/oxford-university-educacic3b3n-popular-y-cambiosocial-en-al-2010.pdf

Riveros, E. (2007). Es posible hablar de calidad en educación popular. REICE: Revista Electrónica Iberoamericana sobre Calidad, Eficacia y Cambio en Educación 5 (5), pp. 244-252

UNESCO.(2000). Desafíos de la Educación. Diez módulos destinados a los responsables de los procesos de transformación educativa. Buenos Aires: IIPE (Instituo Internacional de Planeamiento de la Educación). Recuperado de http://unesdoc.unesco.org/ images/0015/001591/159155s.pdf 